\title{
Efficient Reinforcement Learning of Navigation Strategies in an Autonomous Robot
}

\author{
José del R. Millán \\ Institute for Systems Engineering and Informatics \\ European Commission. Joint Research Centre \\ TP 361. 21020 Ispra (VA). ITALY \\ e-mail: jose.millan@cen.jrc.it \\ Carme Torras \\ Institut de Cibernètica (CSIC-UPC) \\ Diagonal, 647. 08028 Barcelona. SPAIN \\ e-mail: torras@ic.upc.es
}

\begin{abstract}
In this paper we propose a reinforcement learning architecture that allows an autonomous robot to acquire efficient navigation strategies in a few trials. Besides fast learning, the architecture has 3 further appealing features. (1) Since it learns from built-in reflexes, the robot is operational from the very beginning. (2) The robot improves its performance incrementally as it interacts with an initially unknown environment, and it ends up learning to avoid collisions even if its sensors cannot detect the obstacles. This is a definite advantage over non-learning reactive robots. (3) The robot exhibits high tolerance to noisy sensory data and good generalization abilities. All these features make this learning robot's architecture very well suited to real-world applications. We report experimental results obtained with a real mobile robot in an indoor environment that demonstrate the feasibility of this approach.
\end{abstract}

\section{INTRODUCTION}

Efficient navigation is critical for antomomons robots operating in hostile enviromments, which are usually unknown the first time robots face them. This paper deals with the problem of controlling an antomomous mobile robot so that it reaches efficiently a goal location in an unknown indoor enviromment. Instances of the problem where the goal is not inside the perception range of the robot all the time are also considered.

An usial approach to this problem is that of reactive. systems (e.g., [1]). However, basic reactive systems siffer from two shortconings. First, they are difficult to program. Second and nost importiunt, pure reactive controllers may generate innefficient trajectories since they select the next action as a function of the current sensor readings and the robot's perception is limited.

To address this second slortcoming, some approaches

This research has been partially supported by the ESFRIT Basic: Research Action number 7274 combine planning and reaction (e.g., $[2,3]$ ). In contrast to classical planning that acts on a perfect (or sufficiently good) model of the environment, these approaches only require a coarse global map of the environment made out of landmarks. Then, planning takes place at an ahstract level and all the low level details are handled by the reactive component as the robot actually moves. In the case of robots operating in initially unknown environments, this global map can be built from sensory data gathęred either while travelling to the goal (e.g., [2]) or in a previous exploration phase (e.g., [3]).

Global maps are a valuable aid for navigation which must be used when available. When not, we claim that the ardition of learning capabilities to reactive systems is sufficient to allow a robot to generate efficient trajectories after a limited experience. This paper presents experimental results that support this claim: a real autonomous mobile robot equipped with low-resolution sensors learns efficient goal-oriented obstacle-avoidance reactive strategies in a few trials. Moreover, a learning approach like ours even overcomes the first shortcoming of reactive systems. As some researchers have recently shown, the robot programming cost is considerably reduced by letting the robot learn automatically the appropriate navigation strategies (e.g., $[4,5,6,7,8]$ ).

This paper describes the testing of a reinforcement connectionist architecture on a real autonomous mobile robot. A reinforcement-learning robot learns by doing and does not require a teacher who proposes correct actions for all possible situations the robot may find itself in. Instead, the robot simply tries different actions for every situation it encounters and selects the most useful ones as measured by a reinforceme:nt or performance feedback signal. Reinforcement comectionist learning $[9,10,11]$ brings four benefits to antomomons robots. First, this kind of learning robot can inprove its performance continuously and can adapt, itself to new enviromments. Second, the connectionist network does not need to represent explicitly 
all possible situation-action rules as it shows good generalization capabilities. Third, connectionist networks have been shown to deal well with noisy input data, a capability which is essential for any robot working upon information close to the raw sensory datia. Fourtl, comectionist learning rules are well suited to on-line and real-time learning.

In addition to these benefits, the architecture describerl in this paper also overcomes three critical limitations of basic reinforcement connectionist leuming that prevent its application to antonomous rohots operating in the real world. The first and most important limitation is that reinforcement learning mighit require an extremely long time. The main reason is that it is laard to determine rapidly promising parts of the action space where to search for suitable reactions. The second linitation lass to do with the robot's behavior during learning. Practical learning robots should be operational at any moment and, most critically, they shomld avoid catastroplic: failures such as collisions. Finally, the thind limitation concerns the inability of "monolithic" comectionist networks i.e, networks where knowlerlge is clistributed over all the weights - to support incremental learning. In this kind of standard networks, learning a llew rule (or tming an existing one) conld degrarle the knowledge alrearly accquired for other situations.

All important assimption of on appronch is that the robot receives a reinforcement signal after performing every action. Although this assimnption is hard to satisfy in many reinforcement, learning tasks, it is not in the cisse of goal-directerl tasks since the robot can easily evaluate its progress towards the goal at, any nomment. A second assumption of om approacle is that the goal location is known. In particular, the goal location is specified in relative cartesian coordinates with respect to the starting location.

\section{EXPERIMENTAL SETUF'}

The system composed by the robot and the reinforcement comectionist controllel has betul called TESEO. The pliysical robot is a wheelexl cylinulrical nobile platform of the Nomad goo family. It hats these indepenclent motors. The first notor moves the thee wheeds of the rolot together. The second one sterers the wherels together. The third motor rotates the tiuret of the roluot. The robot. has 16 infrared semsors and 16 somar semsors, from which distances to the nearest olstacles can be estinated, and 20 tactile sensors detect collisions. The infrared and somar sensors are evenly placed aromid the perimeter of the turret. Finally, the robot hat a dead-rerkoming system that keeps track of the robot's position and orientation.

The comectionist controller unaps the cmrently perceived situation into a spatially romtimuons action. Then, the controller waits until the robot las finisher to perform the corresponding motor command before conputing the associated reinforcement signal and the next action. We will describe next what the input, ontput and reinforcement signals are.

The input to the connectionist network consists of a vector of 40 components, all of them real numbers in the interval $[0,1]$. The first 32 components correspond to the infrared and somar sensor rearlings. In this case, a vallue close to zero mearis that the corresponding sensor is rletecting a very near obstacle. The remaining 8 components are derived from a virtual sensor that provides the distance between the current and goal robot locations. This sensor is based on the deacl-reckoning system. The 8 conponents correspond to a coarse codification of an inverse exponential function of the virtual sensor reading. The main reason for using this codification scheme is that, since it achieves a sort of interpolation, it offers three theoretical ardvantages, namely a greater robustness, a greater generalization ability and faster learning.

The ontput of the comectionist network consists of a single component that controls directly the steering motor and indirectly the translation and rotation motors. This component is a real number in the interval $[-180,180]$ and determines the direction of travel witl respect to the vector commecting the cimrent and goal robot locations. Once the robot, lans steered the commanded degrees, it translates a fixed distance (10 inclies) and, at the sime time, it rotates its turret in orrer to maintain the front infiared and solnal sensors orienterl toward the gonl.

In this way, a relative codification of botl sensor readings and motor commands with respect, to the goal direction is always maintained. This codification scheme is directly responsible for TESEO's generalization copabilities.

The reinforcenent signal is a real number in the interval $[-3,(1)]$ which nueasures the cost of doing a particnular action in a given situation. The cost of an action is directly derived from the tatsk definition, which is to rench the goal along trajectories that are sufficiently short and, at the same time, have a wirle clearance to the olsstacles. Thus actions incom a cost which depends on both the step clearance mol the stejp length. Concerning the step clearance, the robot is constantly uprating its semsol: rearlings while moving. Thus the step) clealance is the shortest distance measired by any of the sensors while performing the action.

Finally, TESEO is equipperl witl a low-level asynchuro nous emergency rontine to prevent collisions. The robot stops and retracts whenever it detects an obstacle in front of it which is closer than a safety distance. 


\section{EXtensions to BASIC REINForCEMENT LEARning}

TESEO's aim is to learn to perform those actions that optimize the total reinforcement received along the trajectory to the goal. As mentioned in the Introduction, TESEO has been designed to overcome 3 limitations of basic reinforcement learning: slow convergence, lack of incremental improvement, and failure to be operational from the very beginning. The following three aspects of TESEO's architecture ardress these limitations.

First, the connectionist controller is a modular metuork each module codifying a set of similar reaction. rules. That is, these rules map similar sensory inputs into similar actions and, in addition, they have similar long-term consequences. Modularity guarantees that improvements in the response to a given situation will not negatively alter other unrelated reactions.

Second, TESEO explores the action space by concentrating the search around the best actions currently known. The width of the search is determined by a counter-based scheme associated to the modules (see Section IV.B). This exploration technique allows TESEO to avoid experiencing irrelevant actions and to minimize the risk of collisions.

Third, instearl of learning from scratch, TESEO ntilizes a fixed set of basic: reflexes every time its commectionist controller fails to generalize correctly its previous experience to the current situation. The connectionist, controller associates the selected reflex with the perceived sitnation in one step. This new reaction rule is tumed subsecuently through reinforcement learning. Basic: reflexes correspond to previous elemental knowlerge about the task and are codified as simple reactive behaviors [1]. Each reffex selects one of the 16 directions corresponding to the current orientations of the infrared and somar sensors. We latve chosen this fixed set of clirections becalse they are the most informative for the rolost in ternns of olsstacle detection. It is worth noting that, except in simple cases, these reflexes alone do not generute efficient trajectories; they just provide acceptable starting points for the reinforcement learning algorithm to search appropriate actions. Integrating learning and reaction in this way allows TESEO to focus on promising parts of the action space inmediately and to be operational from the very beginning.

\section{Controller Arohitecture}

The comnectionist controller is a morlular two-layer network (Fig. 1). The first layer consists of units with localized receptive fields, which we call exemplars, because each of them represents a proint of the input, space and covers a limited aren aromel this point. The second layer is made of one single stochastic linear unit, the output unit. There exists a full commectivity between the exemplars and the ontput, mit.

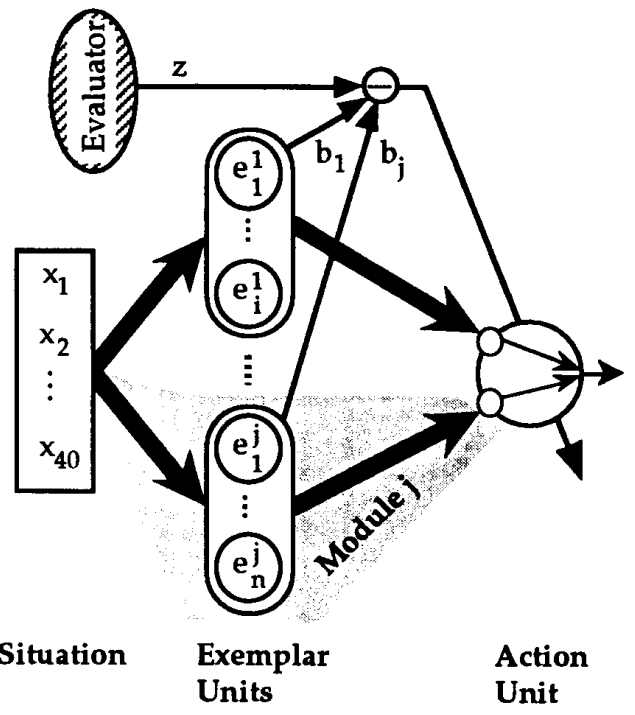

Fig. 1. Controller architext,ure.

\section{A. Exentplars}

The activation level of an exemplar is a value in the interval [0,1], it being 0 if the perceived situation is outside its receptive field, and 1 if the situation corresponds to the point where the exemplar is centered. The $j t h$ module consists of the exemplars $e_{1}^{j}, \ldots, e_{n}^{j}$ and their related links.

All modules respond to each perceived situation, but only the module owning the exemplar with the maximum response propagates the activities of its exemplars to the ontput unit. If no exemplar "matches" the perceived situation -i.e., if the input does not fall in the receptive field of any exemplar-, then the basic reflexes are triggered and the current situation becomes a new exemplar. Section V.A provides more details on this resource-allocating procedure.

The modules are not predefined, bit are created dynamically as TESEO explores its enviromnent. Every module $j$ keejs track of four allaptive values. First, the width of the receptive fielcls of all the exemplars in this module, $d_{j}$. Second, the expected total fiture reinforcement, $b_{j}$, that tlie rolsot will receive if it, uses this module for computing the next action. Third, a counter that records how many times this module has been used withont improving the robot's performance, $c_{j}$. Fourth, the prototypical action the robot slomild normally take whenever the perceived situation is classified into this module, $p a_{j}$. There are as many prototypical actions as reflexes. Thus the first one is the direction of the front infrared and sonar sensors -i.e., a deviation of 0 degrees from the vector connecting the 


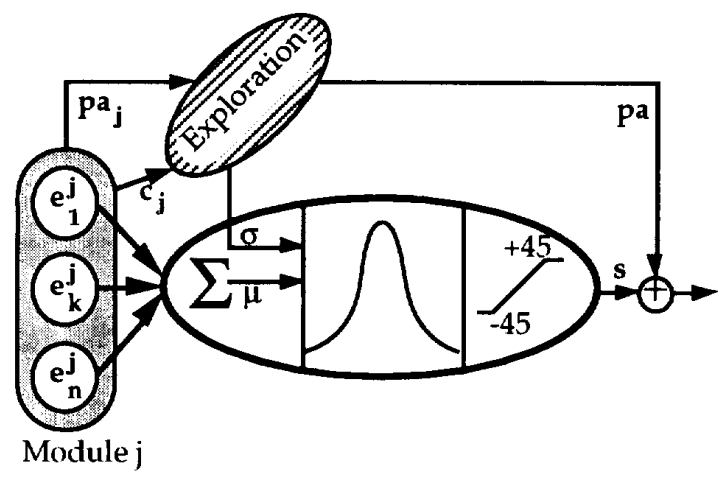

Fig. 2. The output unit.

current and goal robot locations--, the second one is 22.5 regrees, and so on. Sections V.A and VI.B explain low $p a_{j}$ is initially determined and how it evolves, respectively.

After reacting, the enaluator computes the reinforcement signal, $z$, as specified in Section II. Then, if the action was computed throngh the morhle $j$, the difference between $z$ and $b_{j}$ is used for learning (see Section V.D). Only the weights of the links associated to the wimning modile $j$ are modifierl.

\section{B. Output. Unit}

The ontput of the commectionist controller is a prototypical action pa, normally the prototypical action of the winning module, plus a certain variation s that depends on the location of the perceived sitnation in the input sult space dominated by that module (Fig. 2).

In order to find a siditable action for each sitnation throngl reinforcement learning, TESEO needs to explore the action space. However, this exploration is not conducted upon the whole action space, but only aromol the best actions courently known. Since each action is the siun of two components, pa and $s$, the exploration mechlatnism works on each of them separately. This exploration mechanism depends on $c_{j}$, the comber associated to the wimning module.

On the one hand, the explomation medlanism selects pa from the prototypical actions associated to all the modules that classify the perceived situation. That is, if the situation is located in the receptive field of one of the exemplars of the modnles $m$, then $p a_{m}$ is a cancliclate. Assiming if to be the winning morlule, the selection goes as follows. If $c_{j}$ is not divisible by, say, 3 then $p^{\prime} a_{j}$ is chosen. Otherwise, pa is taken to be the prototypical action associated to the morlule $m$ with the best expected total future reinforcenent, $b_{m}$. The hasic idlea behind this exploration mechanism is that the winning module could well benefit from the knowledge of neighboring modules.

On the other hand, the deviation $s$ from $m a$ is computed through a stochastic: process in the interval $\left[-45^{\circ}, 45^{\circ}\right]$. Thus, TESEO will only explore actions between pa and its four neighboring prototypical actions (two to the left and two to the right). The computation of $s$ is done in three steps.

The first step is to rletermine the value of the stochastic: process' parameters. The mean $\mu$ is a weighted sum of the activation levels of the exemplars $e_{1}^{j}, \ldots, e_{n}^{j}$ of the winning module:

$$
\mu=\sum_{k=1}^{n} u r_{k}^{j} a_{k}^{j}
$$

where $w_{k}^{j}$ is the weight associated to the link between $e_{k}^{j}$ and the outpont unit, and $a_{k}^{j}$ is the activation level of $\epsilon_{k}^{j}$. The variance $\sigma$ is proportional to $c_{j}$. This follows from the irleat that the most often the module $j$ is used withont improving TESEO's performance, the higher $\sigma$ must be.

In the second step, the unit calculates its activation level $l$ which is a nommally distributed random variable:

$$
l=N(\mu, \sigma)
$$

In the thirrl step, the unit computes $s$ :

$$
s= \begin{cases}45, & \text { if } l>45 \\ -45, & \text { if } l<-45 \\ l, & \text { otherwise }\end{cases}
$$

\section{Five Learning MEChanisms}

\section{A. Network: Growth}

The first learning medianism makes the controller network grow as a fiuction of the inputs received. Initially, there exist neither exemplars nor, consequently, modules and the resomce-illocating procerlure creates them as they atre needed

As mentioned in Section IV.A, if no exemplar "matches" the perceived situation, then the basic reflexes are triggered and the current sitnation hecomes a new exemplar. That is, both represent the same point of the input spuce. The weight of the link from this exemplin to the ontput unit is initially set to zero and evolves subsequently through reinforement, learning.

The new exemplar is added to one of the existing morlules if its receptive field overlaps the receptive fields of the module's exemplars and the selected reflex is the smo as the mordule's prototylical action. The first condition assimes that every morlule will cover a commected inpout silbipace. 
If any of the two conditions above is not satisfied, then the new exemplar is adcled to a new module. This module consists initially of the exemplar and its associated connections. Concerning the four parameters associated to this new module $f$, they are initially set to the following values: $d_{f}$ equals $0.5, c_{f}$ equals $0, p a_{f}$ is the selected reflex, and $b_{f}$ is estimated on the basis of the distance from the next location to the goal and the distance from the next location to the perceived obstacles in between the robot and the goal.

\section{B. Tuning Exemplars}

The second learning mechanism moves the position of the exemplars $e_{1}^{j}, \ldots, e_{n}^{j}$ of the wimuing module $j$ in order to better cover the input sulsspace dominated hy that, module. That is, the coordinates of the $k^{\text {th }}$ exemplar, $\mathbf{v}_{k}^{j}$, are updated proportionally to how well they matches the input coordinates $\mathbf{x}$ :

$$
\mathbf{v}_{k}^{j}(t+1)=\mathbf{v}_{k}^{j}(t)+\epsilon * a_{k}^{j}(t) *\left[\mathbf{x}(t)-\mathbf{v}_{k}^{j}(t)\right]
$$

where $\epsilon$ is the learning rate. In the experinents reported below, the value of $\epsilon$ is 0.1 .

\section{Improuning Reinforcement Estimates}

The third learning mechanism is related to the update of the future reinforcement estimates $b_{j}$, and it is based on temporal difference (TD) methods [12].

Every value $b_{j}$ is an estimate of the total future reinforcement TESEO will obtain if it performs the best currently known actions that take it from its current location (whose associated observerl situation is classified into the $j^{\text {th }}$ module) to the goal.

Consequently, the value $b_{j}$ of the module $j$ sloould, after learning, be equal to the surn of the cost $z$ of reaching the best next module $i$ phus the value $b_{i}{ }^{1}$ :

$$
b_{j}=\max _{\text {Artioms }}(z)+b_{i} .
$$

In order to iteratively uprlate the values of $b_{j}$, so that finally (5) holds for all of them, we have userl the simplest TD method, i.e. $\operatorname{TD}(0)$ (see [12] for retails). If the situation perceived at time $t$ is classified by module $j$ and, after performing the computed action, the next situation belongs to module $i$ and the reinforcement signal -or cost- is $z(t+1)$, then:

$$
b_{j}(t+1)=b_{j}(t)+\eta *\left[z(t+1)+b_{i}(t)-b_{j}(t)\right] .
$$

The intensity of the modification is controlled by $\eta$, which takes the value 0.75 when TESEO beliaves better

\footnotetext{
1 As described in Section II, $z$ takes negative values. So, minimizing future cost corresponds to maximizing futme reinforcement.
}

than expected, and 0.075 otherwise. The rationale for modifying less intensively $b_{j}$ when $z(t+1)+b_{i}(t)-b_{j}(t)<0$ is that the error in the estimation is probably due to the selection of an action different from the best currently known one for the module $j$.

\section{Weight Update}

The fourth learning mechanism concerns weight modification and uses the classical associative search (AS) [9]. AS uses the estimation given by TD to update the situation-action mapping, which is codified into the connectionist controller:

$$
w_{k}^{j}(t+1)=w_{k}^{j}(t)+\alpha *\left[z(t+1)+b_{i}(t)-b_{j}(t)\right] * \phi_{k}^{j}(t),
$$

where $\alpha$ is the learning rate, and $\phi_{k}^{j}$ is the eligibility factor.

The eligibility factor of a given weight measures how influential that weight was in choosing the action. In our experiments, $\phi_{k}^{j}$ is computed in such a manner that the learning rule corresponds to a gradient ascent mechanism on the expected reinforcement [11]:

$$
\phi_{k}^{j}(t)=\frac{\partial \ln N}{\partial w_{k}^{i}}(t)=a_{k}^{j}(t) \frac{l(t)-\mu(t)}{\sigma^{2}(t)},
$$

where $N$ is the normal distribution function in (2). The weights $u_{k}^{j}$ are modified more intensively in crase of reward -i.e., when TESEO behaves better than expected-- than in case of pencalty. These two values of $\alpha$ are 0.2 and 0.02 , respectively. The aim here is that TESEO maintains the best situation-action rules known so far, while exploring other reaction rules.

\section{Four LEARNing: OPPORTUNITIES}

Let us present now the four occasions in which learning takes place. The first arises during the classification phase, the next two happen after reacting, and the last one takes place when reaching the goal.

\section{A. Unexperienced Situation}

If the perceiverl situation is not classified into one of the existent modules, then the basic reflexes get control of the robot, and the resource-allocating procedure creates a new exemplar which is added either to one of the existing modules or to a new module.

\section{B. Performing urithin Expectations}

If the perceived situation is classified into the module $j$ and $z(t+1)+b_{i}(t)-b_{j}(t) \geq k_{z}$, where $k_{z}$ is a negative constant, then $(i)$ the exemplars of that module are tuned to make them closer to the situation, $(i i)$ the weights 
associated to the connections between the exemplars and the output unit are modified using the AS reinforcement, learning rule, (iii) $b_{j}$ is updated through $\mathrm{TD}(0)$, and (iv) $d_{j}, c_{j}$, and $p a_{j}$ are adapted.

The adaptive parameters are ipolated differently in case of reward than in case of penalty. In case of reward, $a_{j}$ is increased by $0.1, c_{j}$ is initialized to 0 , and if the output of the action unit, $p a+s$, is closer to a prototypical action other than $p a_{j}$, then $p a_{j}$ becomes this new prototypical action. In case of penalty, $c_{j}$ is increased by 1 anrl $d_{j}$ is decreased by 0.1 if it is still greater than a thresloold $k_{d}$.

\section{Performing rather Badly}

If the perceived situation is classified into the module $j$ and $z(t+1)+b_{i}(t)-b_{j}(t)<k_{z}$, then the topology of the network is slightly altererl and $d_{j}$ is decreased by 0.1 if it is still greater than a threslobld $k_{t,}$.

If the total future reinforcement computed after reacting, $z+b_{i}$, is consiclerably worse than the expecterl one, $b_{j}$, this means that the sitnation was inconrectly classified and needs to be clussified into a different morhle.

The resource-allocating procedure creates a new exemplar, $e_{u}$, that has the same comrdinates as the perceived situation, but it does not ard it to any module. The next, time this situation will be facced, $e_{u}$ will be the closest exemplar. Consequently, no mochles will chassify the situation and the basic: reflexes will get control of the robot. Then, the resource-allocating procerlure will ardal $c_{n}$ either to one of the existing morlules or to a new morlule as described in Section V.A.

\section{Reaching the Goal}

Finally, whenever the goal is reached, the value $b_{j}$ of every winning module $j$ along the path to the goal is also updated in reverse chromolongical order. Thls, TESEO needs to store the pairs $\langle j(t), z(t+1)\rangle$ along the corrent, path. This supplementary upelate only accelerates the comvergence of the $b_{j}$ 's, but does not clange their steady values [13].

\section{EXPERIMENTAL RESUlTS}

TESEO's performance laws been tested on a corridor with offices at both sides. The task is to gemerate a slomt but safe trajectory from insicle an office to a point at the end of the corridor. TESEO achieves the target location every time and it never gets lost or trapped into malicionss local maxima. The first time it tries to reach the gonal it relies almost all the time on the hasic reflexes. Essentially, the hasic reflexes make TESEO travel in the direction that (i) is the closest to the comrent, one, (ii) is the safest, and (iii) brings TESEO toward the goal. As illıstrated in Fig. 3, in the first trial, TESEO enters into a clead-end section of

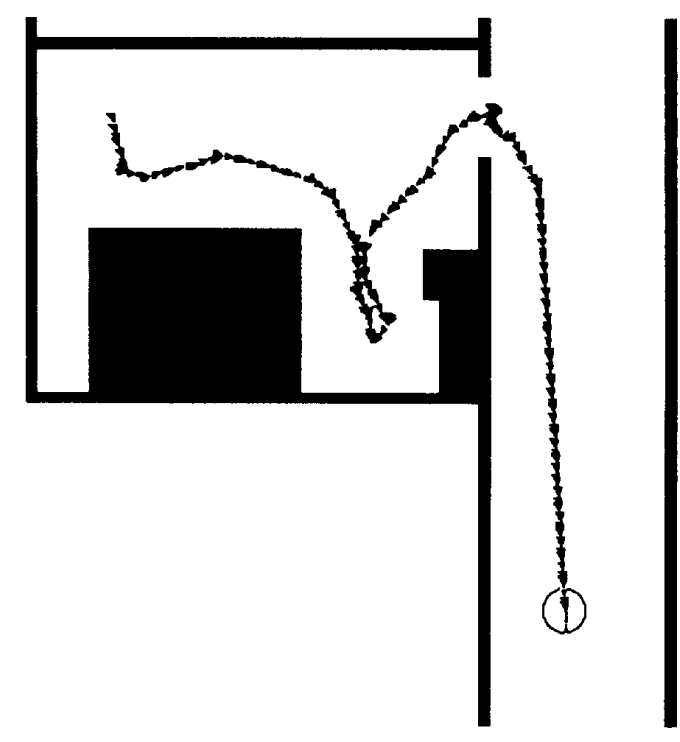

Fig. 3. The enviromment and first tra jectory generated for a starting location within the office. Note that TESEO has some problens in going through the don way.

the office (but it does not get trapped into it) and even it collirles against the door frame becalse its sensors were not able to rletect it. Collisions happened because the frame of the roor is relatively thin and the incident angles of the rays drawn from the sensors were too large resulting in speciular reflections.

Thus this simple taks offers TESEO the opportunity to learn three skills. The first one is to smooth out certain sections of the trajectory generated by the basic reflexes. The second skill is to be abble to avoid dead-ends or, in general, not follow wrong walls. The third and most critical is to avoid olsstacles that its sensors cannot detect.

TESEO learus these three skills very rapidly. It reaches the goal efficiently and withont colliding after travelling 10 times from the starting location to the desired goal (Fig. 4). The total length of the first trajectory is approximately 13 meters while the lengtlı of the trajectory generated after TESEO has learned the suitable sequence of reactions is about, 10 meters. This experiment was rum several times, obtaining similar results.

Conceming the acopisition of the third skill, TESEO associates safe actions to malicions sitnations - made ont of a coarse conlification of the distance to the goal and of mocorrect estimated distances to the obstaclesinmerliately after colliding with mperceived obstacles. Moreover, due to its noise tolerance and generalization (apabilities (see below), TESEO only hits a few times with olstacles that its sensors cannot detect before learning to 

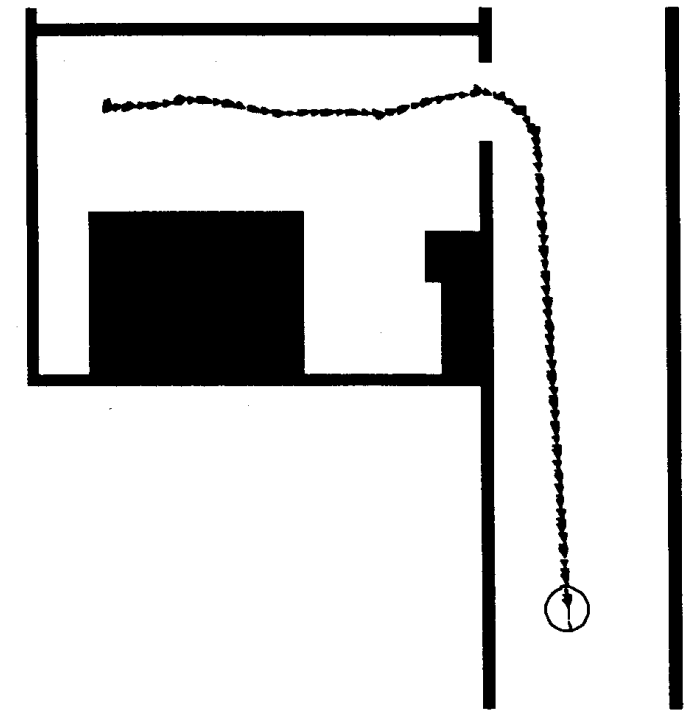

Fig. 4. Trajectory generatex after travelling 10 times to the goal.

avoid them. In addition, the learned navigation strategies have the following features. First, the trajectories are quite smooth even if the basic: reflexes lave not been programmed in this way (it is rather difficult to do it!!) and the reinforcement signal only penalizes long and unsafe paths. Second, the acquired renctions are robust to noisy sensory data. Since sensors are not perfect, TESEO does not perceive the same situations along similar trajectories. However, it is still able to generate suitable actions using only its acquired reactions.

Fig. 5 illustrates instances of the reaction rules learned. For every location considered (little circles) the move to be taken is depicted. Fig. 5 shows that TESEO generates solution paths from any starting location inside tlie room. This simulation experiment, indicates that TESEO exhibits good generalization abilities, since it can handle many more situations than those previously perceived.

Once TESEO has learned efficient, navigation strategies, occasional obstacles are put in the way to the goal. TESEO moves around the obstacles and then it retums to the original, efficient trajectory. In other experiments, the goal is changed. TESEO learns also to navigate to this new goal in a few trials and it is still able to reach the first goal as efficiently as before.

\section{RELATED) WORK}

The robot learning architectule described in this paper shows some resemblancess with several previons works.

In the case of goal-directerl tasks, it is possible to use

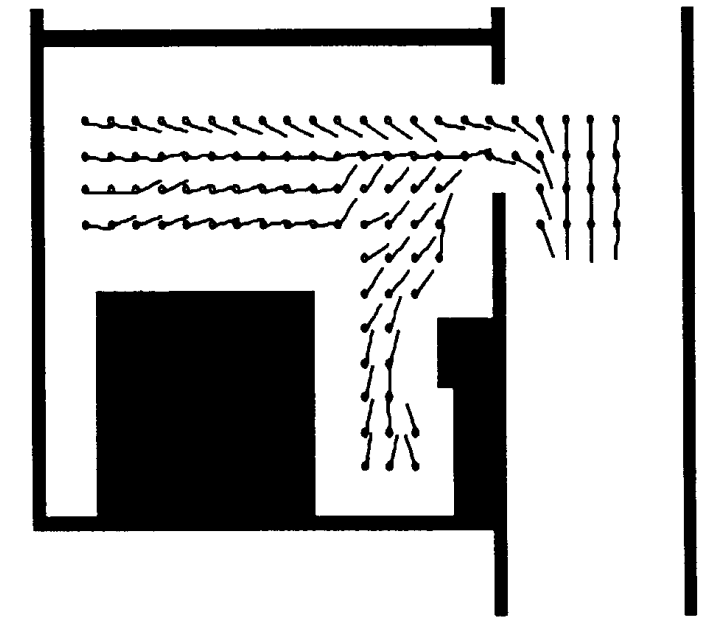

Fig. 5. Generalization abilities: Situation-action rules applied for a sample of locations within the office and the first. part of the corridor.

the future reinforcement associated with situation-action pairs to define a vector field over the environment. Then, the robot will normally perform the action that follows the gradient of the vector field. Seen in this way, our approach is reminiscent of potential field approaches (e.g., [14, 15]). However, our approach differs from them in three fundamental aspects. First, any potential field algorithm relies on predetermining a potential function that will allow the robot to reach the goal efficiently. Contrarily, a reinforcement approach like ours is adaptive in that it starts with an initial and inefficient vector field generated from rough estimates of the future reinforcements and adapts the field to generate efficient trajectories as it converges to the correct estimates through TD methods. Second, the kind of vector fields that can arise in our case are much more varied than those defined by a weighted sum of the potential fields originated by the different obstacles. Third, most of the potential field approaches recuire a global model of the environment while our approach only needs local information obtained from the rohot's sensors. There exist also potential field approaches which only neerl local workspace morlels built out of sensory information (e.g., [14]), but they cannot produce efficient trajectories.

The benefits of letting the robot learn automatically the appropriate reaction rules have recently been emphasized by several anthors. [4] combines reinforcement connectionist learning and teaching to reduce the learning time. In this framework, a human teacher shows the robot several instances of renctive sequences that achieve the task. Then, the robot, learns new reaction rules from these 
examples. The taught reaction rules lielp reinforcement learning by biasing the search for suitable actions toward promising parts of the action space. In our approach, the basic reflexes play the same guidance role, recuiring only a programmer instead of a teacher. [5] use Kohonen maps [16] to split the sensory input space into clusters, and then associate an appropriate action to every cluster through reinforcement learning. Their architecture maps all the situations of a given cluster to a single action, and classifies situations solely by the similarity of their representations. Our architecture classifies situations, first, based on the similarity of their input, representations. But, then, it also incorporates task-specific information for classifying based on the similarity of reinforcements received. In this manner, the input space is split, into consistent clusters since similar future reinforcement corresponds to simular suitable actions for similar sitnlations. [7] integrate induc: tive neural network learning and explanation-based learning. The domain theory is previonsly learued by a set of neural networks, one network for ench riscrete action the robot can perform. Our basic reflexes also represent prior knowlerlge about the task; lowwever, they are much more elemental and are used in a different way

Our approach is also relaterl to the Dyna integrated architectures introduced by [17] and further extended by $[4,18,19]$, among others. Foughly, Dyna uses planning to speed up the acquisition of reaction rules through reinforcement learning. Dyna also learns a global model of the task on which it plans.

\section{SUmmary AND Future WOrK}

We have rlescribed a reinforcement leaming architecture that makes an autonomons mobile robot rapirlly learn efficient navigation strategies in an moknown indoor enviromment. Our robot TESEO not only is operational from the very hegiming and impoves its performance with experience, but also learns to avoirl collisions even when its sensors cannot detect the olsitacles. This is a definite advantage over non-learning reactive robots. TESEO also exhibits incremental learning, high tolerance to noisy sertsory data, and good generalization abilities. All these features make our robot learning arch hitecture very well suited to real-world appliciations.

However, the current implementation of our apponch suffers from one main limitation, namely it recpuires a reliable odometry system that keejs track of the robot's relative position with respect to the goal. Corrently, odomtery is totally hased on dearl-reckoning. In all the experiments we have carried ont so far, deacl-reckoning has proven sufficient to reach the goal. As long as its estimation of the position of the rolot does not differ greatly from the actual one, the commectionist controller is still able to produce correct, actions. But, clearl-reckoning will probably be insufficient in more complicated missions requiring long travels and many turns.

Current work focusses on reliable odometry. The goal is rlesignaterl by a morlulated light beacon and the robot is equipped with sensors especially designed to detect that light. Whenever TESEO detects the goal it uses the beaconing system; otherwise, it relies on dead-reckoning.

\section{REFERENCES}

[1] R.A. Brooks, "A rohust layered control system for a mobile robot," IEEE Journal of Robotics and Automation, vol. 2, pp. $14-23,1986$.

[2] D.P. Miller \& M.G. Slack, "Global symbolic maps from local navigation," Proc. of the ?th National Conf. on Artificial Intelligence, PP. 750-755, 1991

[3] J.H. Connell, "SSS: A hybrid architecture applied to robot, navigation," IEEE Int. Corf. on Robotics and Automation, pp. $2719-2724,1992$.

[4] L.-.J. Lin, "Progranming robots using reinforcenent learning and teaching," Pror. of the !tth National Conf. on Artificial Intelligence, $\mathrm{pl}$. $781-786,1991$.

[5] K. Berns, R. Dilhuann, \& U. Zachnunu, "Reinforcententlearning for the control of an antonomous mobile robot," IEEE/RSJ Int. Coinf. on. Intelligent Robots and Systems, pp. $1808-1815,1902$.

[G] J. del R. Millán \&. C. Torras, "A reinforcement connectionist approach to rolrot path finding in non-maze-like environments," Machine Learning, vol. 8, pp. 363-395, 1992.

[7] T.M. Mitchell \& S.B. Thrun, "Explanation-based neural networks lexuning for roliot control," In C.L. Giles, S.J. Hanson, and J.D. Cowan (eds.), Advances in Neural Information Processing Systems 5, pp. 287-294. San Mateo, CA: Morgan Kaufmann, 19193 .

[8] J. del R. Millán, "Reinforcement learning of goal-directed obstacle-avoidance reaction strategies in an autonomons mobile robot," Robloticis and Autonomens Systems, in press.

[9] A.G. Barto, R.S. Sutton, \& C.W. Anderson, "Nenronlike elements that can solve difficult learning control problems," IEEE Trans. an Systems, Man, and Cybernetics, vol. 13, pr. $835-846,1983$

[10] A.G. Barto, R.S. Sutton, \& C.J. Watkins, "Learning and sequential decision making," Tech. Report 89-95, Dept. of Computer and Information Science, University of Massachusetts, Amlierst, 1989.

[11] R.J. Williams, "Simple statistical gradient-following algorithuss for compertionist reinforcement learning," Machine Learning, vol. 8 , pp. 229-256i, 1992.

[12] R.S. Sutton, "Lentrning to predict thy the methods of termporal differences," Mac:linite Learning, vol. 3, pp. 9-44, 1988.

[13] S. Maliadevan \&. J. Comnell, "Automatic progranming of belhavior-basted rohots using reinforcement learning," Artificial Intelligence, vol. 55, p $, 311-3655,1092$.

[14] J. Borenstein, \& Y. Koren, "Real-time ol stacle avoidance for fast. mobile rohots," IEEE Trans. on Systems, Man, and Cybernetics, vol. 19, pp. 1179-1187, 1989 .

[15] J. Barraquand \& J.C. Latombe, "Rohot motion planning: A clistributerl representation approach," The International Jonrnal of Robotic:s Researchl, vol. 10, pp. (j28-(349, 1991 .

[16] T. Kohonen, Self-Organization and Associative Memory. Second Edition. Berlin: Springer-Verlag, 1988.

[17] R.S. Sutton, "Integgrated architectures for learning, planning, and reacting bassexl on approxinating dynamic programming," Pror: 7th Int. Conf. on Marchine Learning, pp. 216-224, 1990.

[18] L.-J. Lin, "Self-inuproving reactive agents based on reinforceinent leartuing, platning and texaching", Muchine Learning, vol. 8, pp. $2913-321,19992$.

[19] J. Peng \& R.J. Willians, "Efficient learning and planning within the Dyna framework," Adaptive' Beluetior, vol. 1, pp. $437-454,1993$ 